\title{
Spatial and Temporal Varying Thresholds for Cloud Detection in Satellite Imagery
}

\author{
Gary Jedlovec and Stephanie Haines \\ Earth Science Office, VP61 \\ NASA Marshall Space Flight Center \\ 320 Sparkman Drive ,Huntsville, Alabama 35805 \\ gary.jedlovec@nasa.gov
}

\begin{abstract}
A new cloud detection technique has been developed and applied to both geostationary and polar orbiting satellite imagery having channels in the thermal infrared and short wave infrared spectral regions. The bispectral composite threshold (BCT) technique uses only the $11 \square \mathrm{m}$ and $3.9 \square \mathrm{m}$ channels, and composite imagery generated from these channels, in a four-step cloud detection procedure to produce a binary cloud mask at single pixel resolution. A unique aspect of this algorithm is the use of 20-day composites of the $11 \square \mathrm{m}$ and the $11-3.9 \square \mathrm{m}$ channel difference imagery to represent spatially and temporally varying clear-sky thresholds for the bispectral cloud tests. The BCT cloud detection algorithm has been applied to GOES and MODIS data over the continental United States over the last three years with good success. The resulting products have been validated against "truth". datasets (generated by the manual determination of the sky conditions from available satellite imagery) for various seasons from the 2003-2005 periods. The day and night algorithm has been shown to determine the correct sky conditions $80-90 \%$ of the time (on average) over land and ocean areas. Only a small variation in algorithm performance occurs between day-night, Iand-ocean, and between seasons. The algorithm performs least well during the winter season with only $80 \%$ of the sky conditions determined correctly. The algorithm was found to under-determine clouds at night and during times of low sun angle (in geostationary satellite data) and tends to over-determine the presence of clouds during the day, particularly in the summertime. Since the spectral tests use only the short- and long-wave channels common to most multispectral scanners, the application of the BCT technique to a variety of satellite sensors including SEVERI should be straightforward and produce similar performance results.
\end{abstract}

Keywords- Cloud detection, thresholds, GOES, MODIS, SEVERI

\section{NEED FOR CLOUD DETECTION AND MASKING}

The Earth-viewing sensors onboard the current series of Geostationary Operational Environmental Satellites (GOES) provide continuous visible (VIS) and infrared (IR) images of Earth. While the stability of the atmosphere is a key factor affecting regional weather conditions, the presence (or absence) of clouds over a region may influence many aspects of the present and future weather (e.g. visibility, ceilings, insolation, temperatures and changes with time, etc.). The extraction of cloud information from these images is a key component in weather analysis and short-term weather forecasting. The identification of clouds in visible satellite images is relatively straight forward for a trained scientist during the day (although snow and other highly reflective surface features often add complexity to the problem), but this process is substantially more difficult at night when only thermal channels are available.

The automatically detection of clouds in GOES satellite imagery is not a simple task. Poor spatial resolution, changing solar incidence and instrument viewing angles, limited spectral channels, instrument noise, and varying surface properties often limit the success of traditional cloud detection schemes when applied over a large area - both during the day and at night (e.g., Wielicki and Parker 1992; and Dybbroe et al. 2005a). However, the use of data from new high resolution, multispectral instruments such as MODIS has alleviated some of these problems (Platnick et al. 2003; and Ackerman et al. 1998). The linchpin in even the most recent applications is often their dependence on fixed threshold values used in the various individual cloud tests. Often times these threshold values do not represent the variety of atmospheric and surface conditions encountered in the retrieval process. The procedures presented in this paper address these concerns by describing a composite method to develop dynamic thresholds applicable to the local environment. The procedure extends the concept of Dybbroe et al. (2005a) who developed pixel-based dynamic thresholds based on radiative transfer modeling. The current approach utilizes recent satellite data itself to derive spatially and temporally varying thresholds used in the various cloud tests. The approach is demonstrated for GOES Imager data but is applicable to other sensors on geostationary and polar orbiting platforms.

\section{THE BCT APPROACH}

\section{A. Theoretical formulation}

The BCT cloud detection method was developed to address the need for around-the-clock determination of sky conditions over the eastern half of the United States and surrounding ocean regions to support regional climate and short-term forecasting studies and is applicable to other parts of the globe. An underlying principle used in the BCT approach is that the difference between the emissivity of clouds at thermal and at shortwave infrared wavelengths (such as $11.0 \mu \mathrm{m}$ and $3.9 \mu \mathrm{m}$, respectively) varies from that for the surface (land or ocean) and can be detected from channel brightness temperature $\left(\mathrm{T}_{\mathrm{bb}}\right)$ differences. Spectral emissivity varies with both wavelength and surface or cloud type, with 
the emissivity at the shortwave (SW) infrared wavelengths being lower than at the longwave ( $\mathrm{LW}$ ) infrared wavelengths, resulting in lower emission temperatures at the shorter wavelengths. However, during the day reflected solar radiation makes the effective brightness temperatures (sum of emission and reflective components) at the shorter wavelengths greater than the brightness temperatures at the longer wavelengths even though the emissivity is less. Therefore for cloudy pixels, the LW minus SW brightness temperature difference $\left(T_{b b L W}-T_{b b S W}\right)$ has a large negative value during the day and at night a positive value for opaque clouds (thick water clouds and fog) because of the absence of solar radiation, and a negative value for thin ice clouds. Even though the emissivity of thin ice clouds is greater at LW than at SW wavelengths, much of the energy sensed by the satellite comes from the Earth's surface and atmosphere below the cloud and the $3.9 \mu \mathrm{m}$ channel's response to warm sub-pixel temperatures is greater than it is at $11 \mu \mathrm{m}$, resulting in negative difference values both during the day and during the night. Because the difference between shortwave and longwave emissivities is on average smaller for land and water than for clouds, clear pixels will have a small negative temperature difference value during the day and a small negative or positive value at night. Because of these cloud and surface properties, the spatial transition from a clear region to a cloudy region in the satellite image is apparent as a discontinuity in the LW minus SW brightness temperature difference image. Because emissivities vary with cloud type, their effect on the reflected component of the SW channel (due to varying solar input at the surface or cloud top) make the use of these channel differences for cloud detection a challenging problem. The key to the successful detection of clouds having these properties lies in the selection of an appropriate threshold value for the $11-3.9 \mu \mathrm{m}$ difference image which separates cloud-free pixels (where the sensor observes the land) from cloudy pixels. A fixed threshold is not going to produce good results.

A second underlying principle utilized in the BCT cloud mask approach is that the cloud test threshold values can be characterized with composite imagery collected over a specified period of time preceding the current observations. The composites are used to provide spatially and temporally varying clear-sky threshold values to account for changes in surface characteristics that occur over time and between different geographic regions. This approach is similar to that used by Alliss et al. (2000) to determine clear sky background fields from long wave infrared and difference imagery at night. These composite images are created by taking difference images from the same time on the preceding twenty days and forming a composite through some simple arithmetic operation, in this case taking the smallest negative value at each time and pixel from the twenty days of data. Differences in the composite values over a 4 hour period for the same point in the imagery highlight why algorithms which use fixed thresholds may not perform adequately under a variety of conditions.

\section{B. Algorithm description}

The BCT cloud mask method uses multispectral channel differences to contrast clear and cloudy regions. In this application, the $11 \mu \mathrm{m}$ and $3.9 \mu \mathrm{m}$ channels from the GOES Imager are used to produce an hourly difference image (long-wave minus short-wave). Both positive differences, which mainly occur at low sun angles and at night, and negative differences that occur during all times, are preserved in the difference image. From this difference image information, two composite images are created for each hour, which represent the smallest negative (values closest to zero), and the smallest positive difference image values, from the preceding 20-day period. The premise here is that difference image values close to 0 have the highest probability of representing cloud-free pixels. These composite images serve to provide spatially and temporally varying thresholds for the BCT method. An additional 20-day composite image is also generated for each hour using the second warmest longwave $(11 \mu \mathrm{m})$ brightness temperature for each pixel from the 20-day period. (Since no a priori data checks are performed, the elimination of the warmest value avoids bad values due to occasional random noise from affecting the composite). These warm $11 \mu \mathrm{m}$ brightness temperature composite images are assumed to represent warm cloud-free thermal images, one for each time of day.

The 20-day composite images (positive and negative difference images and the warm $11 \mu \mathrm{m}$ images for each hour) used by the BCT cloud mask represent a innovative aspect of the method and provide both spatially and temporally varying clear-sky threshold values for comparison to the observed data. By producing these composites, the BCT approach has the possibility of using a different threshold value from one pixel to the next and therefore location, terrain, sun angle and snow cover are all implicitly taken into account. Single threshold values used by other algorithms must be selected in a conservative way to accommodate the large range of naturally occurring cloud-free values, thereby possibly limiting the effectiveness of the test in their cloud detection approach.

The BCT method uses the 11 and $3.9 \mu \mathrm{m}$ spectral channels in a four-step cloud detection procedure, comprising of two spatial tests and two spectral tests. The two spatial tests are applied to the $11-3.9 \mu \mathrm{m}$ difference images, the first spectral test is also applied to the difference image, and the second spectral test is applied to the $11 \mu \mathrm{m}$ image. The first test subjects each pixel in the $11-3.9 \mu \mathrm{m}$ brightness temperature difference image (DI) to an adjacent pixel test. The variance between pixels DI(i) and DI(i-1) (where the values of $i$ encompass the range of elements in each line of the image) along the scan line in the difference image is computed. If the variance between these adjacent pixels is greater than the variance threshold value, a cloud (edge) is detected. For this test, the threshold value is fixed for all pixels at $7.25 \mathrm{~K}$ and was subjectively determined in earlier work by Haines et al. (2004) from viewing a large number of difference image fields. This test is more successful in identifying the edges of clouds during the day than at night. 
The second step in the BCT method attempts to fill-in between the cloud edges by analyzing the one-dimensional spatial variability of the pixels. The difference between DI(i) and DI(i-1) (i.e., two adjacent pixels) is calculated. For a cloud to be detected, this calculated difference value must be less than the cloudy threshold value if the preceding image location I(i-1) was cloudy (test $2 a$ ), or it must be either less than the negative of the clear threshold value or greater than two thirds of the clear threshold if the preceding image location was clear (2b). In this way the spatial variability in the difference image corresponding to a cloud free surface versus a cloud is considered. Threshold values of 3.0 and 0.0 $\mathrm{K}$, for the clear and cloudy regions, respectively, were derived in a similar fashion as for the first spatial test. The threshold values for these first two tests can be adjusted (tuned) to tweak algorithm performance for particular applications and regions.

The third and fourth steps in the BCT method are used to detect clouds in regions where the first two steps do not detect clouds. The third step utilizes the positive and negative composite images derived for each hour, which represent the smallest positive and negative difference image values, respectively, from the preceding 20 -day period. The minimum difference test ( $3 a$ ) compares the current difference image value to these composite images. A pixel is deemed cloudy if the difference between DI(i) and the smallest positive value is greater than the positive threshold value or if the difference between DI(i) and the smallest negative value is less than the negative of the negative threshold value. The 20day composite positive and negative difference images incorporate spatially varying information for nighttime and daytime cloud determinations separately. Threshold adjustments are added to the composite threshold tests to tune each for improved performance under certain situations in a similar way as Dybbroe et al. (2005b). The fourth and final test in the BCT cloud detection method involves using the LW 11. $\mu \mathrm{m}$ channel information. This IR threshold test uses an hourly 20-day composite of the second warmest thermal infrared channel values at each pixel location. The inclusion of the second warmest pixel reduces the effect of bad values (due to occasional random noise) on the composite. This product is essentially a "warm" cloud free thermal infrared image. A pixel in the observed infrared image is deemed cloudy if its infrared temperature is colder than the composite infrared threshold value (plus its adjustment value) corresponding to its location and time period.

The fixed input threshold values (for tests 1 and 2) and the adjustment values added to the composite values (used in tests 3 and 4) are provided in Table 1. Typical ranges in the composite image values for GOES data used in tests 3 and 4 are also presented. For the positive difference image test, observed difference image values which are smaller that the composite plus the threshold value $(2.5 \mathrm{~K})$ are deemed clear. For the negative threshold test, observed difference values which are greater than the composite value minus the threshold value (4) are deemed to be clear. For the IR threshold test (4), an observed IR temperature needs to be 18.5 $\mathrm{K}$ cooler than the clear-sky composite IR temperature for a cloud to be detected, which corresponds to IR temperatures in the range $230 \mathrm{~K}-295 \mathrm{~K}$ (approximately) depending on location, time of day and season. For example, at midday in summertime, the clear-sky value may be as warm as $305 \mathrm{~K}$, and any pixels cooler than $286.5 \mathrm{~K}$ would be determined to be clouds, but at night during wintertime, the clear-sky value may be $265 \mathrm{~K}$, more than $20 \mathrm{~K}$ cooler than the maximum cloud temperature during the summertime example, and clouds would have to be $246.5 \mathrm{~K}$ or cooler. Without the use of the composite imagery to define spatially and temporally varying thresholds, a single temperature threshold would not represent the appropriate test cutoffs for accurate cloud detection.

Table 1. Fixed threshold values for the spatial tests and adjustment values for the spectral tests for the GOES Imager.

\begin{tabular}{|c|c|c|c|}
\hline Test & $\begin{array}{c}\text { Threshold } \\
\text { name }\end{array}$ & Value & $\begin{array}{c}\text { Approximate } \\
\text { Composite Range } \\
\text { (Clear sky values) }\end{array}$ \\
\hline $\begin{array}{c}\text { 1-Adjacent } \\
\text { Pixel }\end{array}$ & variance & 7.25 & NA \\
\hline $\begin{array}{c}2-1-D \\
\text { Variability }\end{array}$ & clear & 3.0 & NA \\
\hline $\begin{array}{c}2-1-D \\
\text { Variability }\end{array}$ & cloud & 0.0 & NA \\
\hline $\begin{array}{c}3-\text { Minimum } \\
\text { Difference }\end{array}$ & positive & 2.5 & $0-5$ \\
\hline $\begin{array}{c}3-\text { Minimum } \\
\text { Difference }\end{array}$ & negative & 4.0 & $-15-0$ \\
\hline $\begin{array}{c}4-\text { Infrared } \\
\text { Threshold }\end{array}$ & infrared & 18.5 & $250-315 \mathrm{~K}$ \\
\hline
\end{tabular}

\section{VALIDATION}

It is important to know the accuracy of any cloud mask in detecting clouds and the times or situations when the mask may not perform as well. To assess this for the BCT cloud detection method applied to GOES Imager data, the BCT approach was validated by comparing a subjective determination of the sky conditions from GOES visible and infrared satellite imagery to the cloud mask produced by the algorithm. Thirty boxes, measuring approximately $30 \mathrm{~km}$ by $30 \mathrm{~km}$ each, were selected over the eastern two-thirds of the United States including the Atlantic Ocean and the Gulf of Mexico to serve as validation locations. The locations represent a variety of surface and terrain conditions including land, coastal regions, and ocean. For each box, a trained satellite meteorologist determined the sky condition to be either clear ( $10 \%$ or less cloud cover), cloudy ( $90 \%$ or more cloud cover), or partly cloudy (between $10 \%$ and $90 \%$ cloud cover), and then compared this to the cloud mask. The use of a satellite meteorologist to produce a "truth" dataset is consistent with methods of Merchant et al. (2005). The 
comparison between the observations and the cloud mask had three possible outcomes, 1) the cloud mask algorithm correctly determined the sky conditions as clear ( $Z$, clear correct), cloudy or partly cloudy ( $\mathrm{H}$, hits or correct cloudy), 2) the cloud mask algorithm over-determined clouds by labeling the pixels within the box as partly cloudy or cloudy and the observed state of scene was clear or partly cloudy $(\mathrm{F}$, overdetermination or false alarms), and 3 ) the cloud mask algorithm under-determined clouds by labeling the pixels in the box as clear or partly cloudy with the observations indicating a partly cloudy or totally cloudy scene (U, underdetermination of clouds or misses).

Sky conditions were determined by examining GOES-12 visible (daytime only) and IR imagery (day and night). By viewing the GOES imagery at the same resolution, projection, viewing angle and coverage as the cloud mask, validation results were produced with a high level of confidence. Some problems did arise with this validation method. Human bias (of the satellite meteorologist) in the subjective determination of cloud or no cloud within a given box, especially at night when only infrared imagery is available was noted in the results. To help overcome this problem, loops of the imagery were generated to view the movement of clouds with time, allowing for the discrimination between snow and clouds and between warm clouds and the surface. Although looping the imagery resolved the sky conditions for many of the times and locations, there were cases when the observer was unsure of the sky conditions, and these were labeled as uncertain. The rejection of these points from consideration is consistent with that of Merchant et al. (2005a and $b$ ) in their validation studies. To further reduce the influence of human error or bias, several case periods were studied to provide a large data pool, and several satellite meteorologists with different satellite experiences were used. The results from the different experts were averaged together.

The BCT cloud algorithm was validated for the months of October 2003, January 2004, April 2004 and June 2004 , representing the different seasons and associated surface and weather conditions over the eastern half of the United States. During this time, the GOES-12 satellite routinely collected Imager data under nominal collection schedules which included coverage of the continental U.S. and surrounding ocean area. For each day in the dataset, four different times were studied, corresponding to nighttime and daytime, and approximations of sunrise and sunset. Sunrise and sunset times where chosen because of the known difficulty in detecting clouds in satellite imagery at these times. During each season one or two of the four weeks were validated by two satellite meteorologists, providing a total of 20 weeks of data. With 20 weeks of observations, four times a day, and 30 boxes, there was a possibility of 16,800 observations, minus three missing times and 35 uncertain points (only $0.2 \%$ of the total observations), the total number of observations made was 16,675 .

\section{RESULTS}

Table 2 provides the overall results of the BCT GOES cloud mask algorithm validation and summarizes the overall performance for the combined seasons, regions and times used in this study. The BCT cloud mask algorithm correctly determined sky conditions $87.6 \%$ (14613 boxes) of the time. This performance exceeds the $71 \%$ reported by Schreiner et al. (2001) for GOES-8-10 Sounder data. Of these correct determinations, $44.2 \%$ (6466 boxes) were from clear regions and $55.8 \%$ ( 8147 boxes) from cloudy regions. Of the $12.4 \%$ (2062) incorrect determinations, only $3.4 \%$ (570) of the total placed clouds in regions determined clear in the visible and/or infrared imagery (over-determination), and $9.0 \%(1492)$ of the total missed clouds that were seen in the imagery (under-determination). These numbers correspond to a hit rate, HR (number of correct cloud points normalized by the total number of cloudy points) $84.5 \%$. The overall false alarm rate, FAR (number of false cloudy points normalized by the number of clear points) for the BCT algorithm $8.1 \%$. The resulting skill score (HR - FAR) is 76.4 These generalized results indicate that the $\mathrm{BCT}$ algorithm has a tendency to under-determine clouds, however, as the conference presentation will show, the performance of the algorithm is dependent on both the season and the time of day.

Table 2. Overall performance results of the BCT cloud mask algorithm applied to GOES-12 Imager data. The statistics indicate a $F A R=8.1, H R=84.5$, and a $S S=76.4$

\begin{tabular}{|c|c|c|c|c|c|c|c|}
\hline & OBS & C & I & F & U & Z & H \\
\hline$\#$ & 16675 & 14613 & 2062 & 570 & 1492 & 6466 & 8147 \\
\hline$\%$ & 100 & 87.6 & 12.4 & 3.4 & 8.9 & 44.2 & 55.8 \\
\hline
\end{tabular}

\section{REFERENCES}

[1] Ackerman, S.A., K. I. Strabala, W.P. Menzel, R. A. Frey, C.C. Moeller, and L. E. Gumley, 1998: Discriminating clear-sky from clouds with MODIS, $J$. Geophys. Res. 103, 32,141-32,158.

[2] Alliss, R. J., M. E. Loftus, D. Apling, and J. Lefever, 2000: The development of clond retrieval algorithms applied to GOES digital data. Preprints, 10th Conference on Satellite Meteorology and Oceanography, Long Beach, AMS, 330-333.

[3] Dybbroe, A., K-G. Karlsson, and A. Thoss, 2005b: NWCSAF AVHRR cloud detection and analysis using dynamic thresholds and radiative transfer modeling. Part II: Tuning and validation. J. Appl,. Meteor. 44, 55-71.

[4] Haines, S. L., R. J. Suggs, and G. J. Jedlovec, 2004: The GOES Product Generation System, NASA Technical Memorandum 2004-213286, Marshall Space Flight Center, Alabama, 50 pgs.

[5] Platnick, S., M. D. King, S. A. Ackerman, W. P. Menzel, B. A. Baum, C. Riedl, and R. A. Frey, 2003: The MODIS cloud products: Algorithms and examples from Terra. IEEE Transactions on Geoscience and Remote Sensing, Aqua Special Issue, 41, 459-473. 